\title{
Melhoramento e estrutura populacional em bubalinos da raça Mediterrâneo no Brasil
}

\author{
Carlos Henrique Mendes Malhado(1), Alcides de Amorim Ramos (2), Paulo Luiz Souza Carneiro(1), \\ Danielle Maria Machado Ribeiro Azevedo ${ }^{(3)}$, Raimundo Martins Filho ${ }^{(4)}$ e Julio César de Souza ${ }^{(5)}$
}

\begin{abstract}
(1)Universidade Estadual do Sudoeste da Bahia, Departamento de Ciências Biológicas, Av. José Moreira Sobrinho, $s / n=$ o, Jequiezinho, CEP 45200-000 Jequié, BA. E-mail: carlosmalhado@gmail.com, plscarneiro@gmail.com (2)Universidade Estadual Paulista, Faculdade de Medicina Veterinária e Zootecnia, Departamento de Produção e Exploração Animal, Fazenda Lageado, Caixa Postal 560, CEP 18618-000 Botucatu, SP. E-mail: aaramos@fca.unesp.br ${ }^{(3)}$ Embrapa Meio-Norte, Unidade de Execução de Pesquisas de Parnaíba, Caixa Postal 341, CEP 64200-970 Parnaíba, PI. E-mail: azevedo@cpamn.embrapa.br (4)Universidade para o Desenvolvimento do Estado e da Região do Pantanal, Campus I Miguel Couto, CEP 79003-010 Campo Grande, MS. E-mail: rmartinsfilho@yahoo.com.br (5)Universidade Federal do Paraná, Campus de Palotina, Rua Pioneiros, no 2153, Centro, CEP 85950-000 Palotina, PR. E-mail:jcs@ufpr.br
\end{abstract}

\begin{abstract}
Resumo - O objetivo deste trabalho foi avaliar a estrutura populacional e os progressos genéticos, fenotípicos e ambientais de características de desenvolvimento ponderal, em bubalinos da raça Mediterrâneo. Foram utilizadas informações dos pesos ajustados aos 205, 365 e 550 dias de idade, de 6.243 bubalinos nascidos no período de 1974 a 2003, provenientes de quatro fazendas. As estimativas dos coeficientes de herdabilidade foram altas para os pesos ajustados nas três idades. Os rebanhos apresentaram ganho genético positivo quanto a essas três características, porém, aquém do possível, provavelmente em razão da seleção baseada somente no fenótipo, realizada pelos produtores. Para aumento nos ganhos genéticos, são necessários a redução do intervalo de geração, o aumento do tamanho efetivo, o uso de reprodutores avaliados com base nos valores genéticos preditos pelo BLUP e o controle dos acasalamentos de animais aparentados.
\end{abstract}

Termos para indexação: Bubalus bubalis, endogamia, ganho genético, intervalo de geração, tamanho efetivo.

\section{Improvement and population structure of Mediterranean water buffaloes raised in Brazil}

\begin{abstract}
The objective of this work was to evaluate the population structure as well as the improvement of genetic, phenotypic, and environmental effects on growth development of Mediterranean water buffaloes, raised in Brazil. Information on weight adjusted at 205, 365, and 550 days of age of 6,243 buffaloes from four farms, which were born between 1974 and 2003, were used. Heritability coefficients for weight were high at the three ages. The herds presented a positive genetic gain in the three analyzed traits, although lower than expected, what was likely to be caused by empirical phenotype-based selection used by the local breeders. In order to improve genetic gains, it is necessary to decrease the generation interval, to increase the effective size, to use reproducers whose genetic values have been previously evaluated by BLUP, and to avoid mating between related animals.
\end{abstract}

Index terms: Bubalus bubalis, inbreeding, genetic gain, generation interval, effective size.

\section{Introdução}

Há muitas décadas, os bubalinos vêm sendo criados no Brasil e, atualmente, existem cerca de 2,8 milhões de bubalinos distribuídos por todos os estados brasileiros (Ramos et al., 2006). Essa espécie tem apresentado grande expansão em território brasileiro, com crescimento superior ao dos bovinos (Malhado et al., 2007).
Os bubalinos de corte possuem notável rusticidade, capacidade de conquistar espaços muitas vezes inacessíveis ao homem e a outras espécies, resistência a endo e ectoparasitas e a diversas doenças infecto-contagiosas. Além disso, seus produtos (carne e leite) atendem a todas as exigências estabelecidas para o consumo humano, na comercialização e industrialização (Malhado, 2005). 
Entretanto, ao contrário do que ocorre com os bovinos, não existe um programa intenso de melhoramento genético desta espécie, e as iniciativas existentes nesse sentido estão associadas à tenacidade de poucos pesquisadores e criadores.

A fim de viabilizar os programas de melhoramento genético, é imprescindível conhecer os diferentes fatores que interferem potencialmente na seleção e no progresso genético, como por exemplo, tamanho efetivo, intervalo de gerações e variabilidade genética.

Muir (2000) relata que, entre os principais fatores que afetam a resposta à seleção, além do tamanho efetivo da população, estão a precisão e a intensidade de seleção. Freqüientemente, estudos indicam que a resposta ótima à seleção pode ser obtida maximizando-se estes fatores. Entretanto, nem todos os fatores podem ser maximizados simultaneamente. O aumento na intensidade de seleção, por exemplo, provoca decréscimo no tamanho efetivo da população e, conseqüentemente, na resposta à seleção.

Carneiro et al. (2006) citam que a seleção, com base nos valores genéticos obtidos pelos procedimentos estatísticos, baseados na metodologia dos modelos mistos (BLUP), pode conduzir ao rápido aumento na taxa de endogamia e na percentagem de alelos fixados desfavoravelmente, especialmente em populações de pequeno tamanho efetivo e para características de baixa herdabilidade. Nesse trabalho, os autores frisaram que, em programas de melhoramento que utilizam pequenas populações sob seleção, os resultados podem ser influenciados pela deriva genética, o que pode ocasionar variações nos ganhos genéticos.

Diversos estudos no Brasil, com bovinos das raças zebuínas, vêm evidenciando pequenos progressos genéticos para características de desenvolvimento ponderal (Oliveira et al., 1995; Euclides Filho et al., 2000; Ferraz Filho et al., 2002). Entretanto, esses estudos não abordaram a estrutura populacional dos rebanhos.

O monitoramento do progresso genético deve ser uma preocupação para toda e qualquer raça (Euclides Filho et al., 2000). De acordo com esses pesquisadores, o conhecimento da evolução genética de uma população tem importância não só para proceder aos ajustes que se fizerem necessários, mas também para que se avalie o resultado do programa de seleção que vem sendo adotado.

O objetivo deste trabalho foi avaliar a estrutura populacional e o progresso genético, fenotípico e ambiental das características de desenvolvimento ponderal em bubalinos da raça Mediterrâneo, criados no Brasil.

\section{Material e Métodos}

A base de dados utilizada pertence ao Programa de Melhoramento Genético de Bubalinos (Ramos et al., 2002). Foram utilizadas informações de 6.243 bubalinos da raça Mediterrâneo nascidos no período de 1974 a 2003, em quatro fazendas localizadas nos estados do Rio Grande do Sul, Paraná, São Paulo e Bahia. Na Tabela 1 é apresentada a estrutura do conjunto de dados utilizados para cada característica.

Para obter as estimativas das (co)variâncias e dos valores genéticos, empregou-se a metodologia da Máxima Verossimilhança Restrita Livre de Derivada, por meio de modelos animais unicaracterística, usando o aplicativo Multiple Traits Derivate Free Restrict Maximum Likelihood (Boldman et al., 1995).

Para os pesos ajustados aos 205 (P205), 365 (P365) e 550 (P550) dias de idade, foram utilizados modelos que incluíram os efeitos aleatórios genéticos, direto e materno, e de ambiente permanente, além dos efeitos fixos de grupo contemporâneo e grupo genético, admitindo a covariância entre os efeitos direto e materno como nula $\left(\sigma_{\mathrm{am}}=0\right)$. Os grupos de contemporâneos foram constituídos de animais do mesmo sexo, época (estação e ano de nascimento) e fazenda. Grupos de contemporâneos com menos de cinco animais foram excluídos das análises.

As estimativas das tendências genéticas, fenotípicas e ambientais para as características foram obtidas pela regressão da média da variável dependente (valores genéticos, pesos observados, solução dos grupos de contemporâneos) sobre o ano de nascimento dos animais, por meio do SAS Institute (1999).

Utilizou-se a regressão não-paramétrica por meio de polinômios articulados (spline) para descrever as tendências observadas nos gráficos.

O estimador Spline tem a forma:

$$
S(\lambda)=\frac{1}{3} \sum_{i=1}^{n}\left(y_{i}-\hat{f}_{\lambda}(x)\right)^{2}+\lambda \int_{-\infty}^{\infty}\left(\hat{f}_{\lambda}(x)\right)^{2} d x
$$

em que $\hat{f}_{\lambda}$ pertence ao conjunto das funções

Tabela 1. Estrutura de dados no arquivo estudado para os pesos ajustados aos 205 (P205), 365 (P365) e 550 (P550) dias de idade, em bubalinos da raça Mediterrâneo.

\begin{tabular}{lrrr}
\hline Característica & P205 & P365 & \multicolumn{1}{c}{ P550 } \\
\hline Número total de registros & 4.930 & 4.930 & 4.930 \\
Número de registros utilizados & 4.361 & 4.472 & 798 \\
Número de touros & 84 & 84 & 34 \\
Números de vacas & 1.753 & 1.769 & 498 \\
Número de grupos contemporâneos & 430 & 439 & 152 \\
Número de animais na matriz de parentesco & 6.243 & 6.243 & 6.243 \\
\hline
\end{tabular}


continuadamente diferenciadas, com derivadas de segunda ordem de integráveis quadradas, onde $\lambda$ é uma constante positiva. O estimador resultante da minimização $S(\lambda)$ é chamado de estimador de alisamento Spline. Esse estimador ajusta um polinômio cúbico em cada intervalo entre pontos. Para cada ponto $\mathrm{x}_{\mathrm{i}}$, a curva e suas primeiras duas derivadas são contínuas. $\mathrm{O} \lambda$ é o parâmetro que controla a troca entre a bondade e o alisamento do ajuste. Seleciona-se um parâmetro de alisamento $\lambda$ por especificar uma constante c na fórmula $\lambda$ - $(\mathrm{Q} / 10) 3 \mathrm{c}$, em que Q é o intervalo interquartílico. Essa fórmula faz com que c seja independente da unidade de $\mathrm{x}$.

O intervalo médio de gerações das quatro passagens gaméticas foi estimado a partir dos passos: pai-filho, paifilha, mãe-filho e mãe-filha através do software ENDOG (Gutierrez \& Goyache, 2005).

Também com o ENDOG, estimou-se o tamanho efetivo da população $\left(\mathrm{N}_{\mathrm{e}}\right)$, por meio da taxa de variação dos coeficientes médios de endogamia entre gerações sucessivas, conforme a fórmula:

$\Delta \mathrm{F}=\frac{\mathrm{F}_{\mathrm{t}}-\mathrm{F}_{\mathrm{t}-1}}{1-\mathrm{F}_{\mathrm{t}-1}}$, com rearranjos: $\mathrm{N}_{\mathrm{e}}=\frac{1}{2 \Delta \mathrm{F}}$, em que: $F_{t}$ é o coeficiente médio de endogamia, estimado na geração atual; $F_{t-1}$ é o coeficiente médio de endogamia estimado na geração anterior.

\section{Resultados e Discussão}

As médias e os desvios-padrão para os pesos ajustados aos 205 (P205), 365 (P365) e 550 (P550) dias de idade foram 196, $0 \pm 33,5 ; 301,6 \pm 58,7$ e 427,25 $\pm 89,0 \mathrm{~kg}$, respectivamente. Jorge et al. (2005), ao estudar bubalinos da raça Murrah, no Estado de São Paulo, relataram médias de $170,2 \pm 8,9,251,8 \pm 31,8$ e $322,8 \pm 41,9 \mathrm{~kg}$ para os pesos ajustados aos $240,365 \mathrm{e}$ 550 dias, respectivamente.

As estimativas do intervalo de gerações para as quatro passagens gaméticas foram 11,2 $\pm 3,7$ (pai-filho), 12,1 $\pm 3,9$ (pai-filha), 9,6 $\pm 4,8$ (mãe-filho), 10,6 $\pm 4,6$ anos (mãe-filha). O intervalo de gerações médio foi 11,3 $\pm 4,3$ anos. Este valor é considerado alto, quando comparado ao intervalo de gerações de bovinos. Faria et al. (2002) e Vercesi Filho et al. (2002) relataram médias de 7,2 e 7,1 (pai-filho), 7,2 e 7,2 (pai-filha), 7,0 e 7,2 (mãe-filho) e 6,9 e 7,1 anos (mãefilha) para animais registrados das raças Nelore Mocho e Tabapuã, respectivamente.
Os bubalinos são reconhecidos pela sua longa vida produtiva e reprodutiva e a necessidade de crescimento do rebanho ao longo dos anos permite que alguns criadores mantenham os reprodutores e matrizes por maior período no rebanho, o que ocasiona incremento do intervalo de geração.

A utilização de reprodutores por tempo limitado proporciona redução no intervalo de gerações, além da possível redução na variância do tamanho de família que tende a minimizar decréscimos no tamanho efetivo da população (Faria et al., 2001).

A otimização do intervalo médio de gerações é fundamental em programas de melhoramento genético, pois intervalos muito grandes diminuem o ganho genético anual para as características selecionadas, o que leva a menor retorno econômico do programa (Faria et al., 2001).

O coeficiente médio de endogamia da população aumentou com o decorrer dos anos, passando de $0,6 \%$ na primeira geração para 1,9\% na segunda. Esse resultado pode ser, em parte, explicado pelo tamanho efetivo da população que era de 83,4 na primeira geração e passou para 38,1 na segunda.

Cunha et al. (2006) frisaram que o decréscimo do tamanho efetivo pode estar associado ao aumento do nível de endogamia. De acordo com os autores, isso ocorre, principalmente, em razão do uso intensivo de alguns poucos touros melhoradores nos rebanhos e do aumento observado da variância do número de progênies por reprodutor em gerações sucessivas.

O principal fato sugestivo para a explicação do aumento da endogamia e diminuição do tamanho efetivo nos bubalinos estudados é o aumento, nos últimos anos, da utilização da inseminação artificial. Apesar das vantagens dessa técnica, quando utilizada de forma não adequada, pode fazer com que alguns reprodutores sejam utilizados intensivamente, o que reduz o tamanho efetivo e aumenta o acasalamento de indivíduos aparentados nos rebanhos.

Goddard \& Smith (1990) sugeriram tamanho efetivo mínimo de 40 animais por geração, para maximizar o retorno econômico, enquanto Meuwissen \& Woollians (1994) recomendaram valores entre 31 e 250 , para prevenir decréscimos no valor adaptativo. 
Os componentes de (co)variância e os coeficientes de herdabilidades, direta e materna, são apresentados na Tabela 2. Os coeficientes de herdabilidades, direta e materna, para o peso aos 205 dias de idade foram $0,42 \pm 0,06$ e $0,05 \pm 0,03$, respectivamente. Lôbo et al. (2000), com base em 78 trabalhos sobre bovinos, observaram herdabilidade média de 0,30 para o efeito direto. Já Gianotti et al. (2005) relataram herdabilidade de 0,23 , ao revisarem 331 trabalhos, com bovinos.

Os coeficientes de herdabilidades (direto) para P365 e P550 foram $0,41 \pm 0,06$ e $0,50 \pm 0,15$, respectivamente. Lôbo et al. (2000) e Gianotti et al. (2005) relataram herdabilidade média em bovinos de 0,37 e 0,27 para $\mathrm{P} 365$, e 0,35 e 0,31 para P550, respectivamente.

Os valores dos coeficientes de herdabilidades, para os efeitos diretos, estimados para as três características, são altos e indicam alta variabilidade genética aditiva direta, evidenciando a oportunidade de ganho genético com a seleção.

Para o peso aos 205 dias de idade, a tendência genética (regressão linear) do efeito direto foi significativa $(\mathrm{p}<0,01)$ e igual a $0,37 \mathrm{~kg} \mathrm{ano}^{-1}$, o que representa progresso genético de $4,4 \mathrm{~kg}$, nos 11 anos analisados (Figura 1 A). Por sua vez, a tendência genética (regressão linear) materna não foi significativa $(\mathrm{p}>0,05)$.

As tendências genéticas (regressão linear) para os pesos aos 365 e 550 dias de idade, para o efeito direto, foram significativas $(\mathrm{p}<0,01)$ e iguais a $0,39 \mathrm{e} 0,65 \mathrm{~kg}^{2} \mathrm{ano}^{-1}$, respectivamente, com progresso genético de 4,7 e $7,8 \mathrm{~kg}$ no período (Figura $1 \mathrm{~B}$ e C). Os ganhos genéticos no período correspondem aos incrementos de 1,55 e 1,82\% nos pesos aos 365 e 550 dias de idade, respectivamente. As tendências genéticas maternas para as características na fase pós-desmama (P365 e P550) não foram significativas $(\mathrm{p}>0,05)$.

Tabela 2. Estimativas dos componentes de (co)variância e herdabilidades para os pesos ajustados aos 205 (P205), 365 (P365) e 550 (P550) dias de idade, em bubalinos da raça Mediterrâneo $^{(1)}$.

\begin{tabular}{|c|c|c|c|c|c|}
\hline Variável & $\sigma_{a}^{2}$ & $\sigma_{\mathrm{m}}^{2}$ & $\sigma_{\text {ep }}^{2}$ & $\overline{\sigma_{p}^{2}}$ & $\mathrm{~h}_{\mathrm{m}}^{2}$ \\
\hline P205 & 311,17 & 36,22 & 344,66 & 747,27 & $0,42 \pm 0,060,05 \pm 0,03$ \\
\hline P365 & 807,56 & 99,81 & $24,81 \quad 1.019,58$ & $1.951,18$ & $0,41 \pm 0,060,05 \pm 0,03$ \\
\hline P550 & $1.849,34$ & 393,77 & $177,501.264,67$ & $3.686,30$ & $0,50 \pm 0,150,11 \pm 0,08$ \\
\hline
\end{tabular}
herdabilidade direta e herdabilidade materna.
Na Tabela 3, estão representados os ganhos genéticos específicos para os quatro rebanhos. Apenas um rebanho obteve ganho genético linear positivo e significativo nas três características. Deve ser ressaltado que dos três rebanhos considerados multiplicadores, istoé, vendedores de reprodutores, dois não estão apresentando ganhos genéticos nas características estudadas (Tabela 4). Até 2002, não existia avaliação genética em bubalinos.
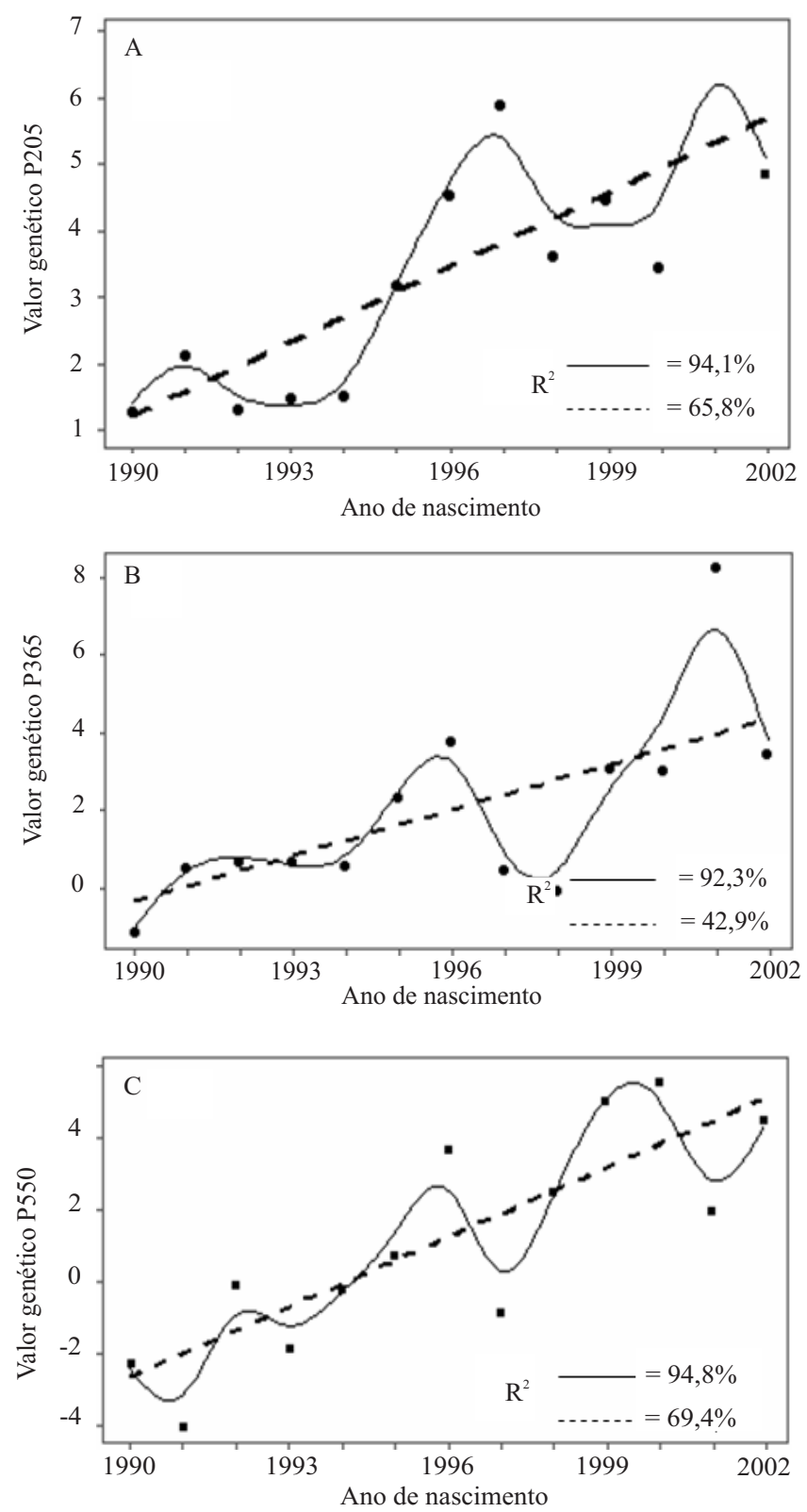

Figura 1. Tendências genéticas do efeito direto para os pesos ajustados aos 205 (A), 365 (B) e 550 (C) dias de idade, em bovinos da raça Mediterrâneo. 
Tabela 3. Ganho genético $(\Delta \mathrm{G})$ em quatro rebanhos de bubalinos da raça Mediterrâneo.

\begin{tabular}{|c|c|c|c|c|c|c|}
\hline \multirow[t]{2}{*}{ Rebanho } & \multicolumn{2}{|c|}{ P205 } & \multicolumn{2}{|c|}{ P365 } & \multicolumn{2}{|c|}{ P550 } \\
\hline & $\Delta \mathrm{G} /$ ano $(\mathrm{kg})$ & $\mathrm{R}^{2}(\%)$ & $\Delta \mathrm{G} /$ ano $(\mathrm{kg})$ & $\mathrm{R}^{2}(\%)$ & $\Delta \mathrm{G} /$ ano $(\mathrm{kg})$ & $\mathrm{R}^{2}(\%)$ \\
\hline$\overline{\mathrm{A}}$ & $1,02 * * *$ & 79,2 & $0,79 * * *$ & 85,1 & $0,86 * * *$ & 67,2 \\
\hline B & $0,15^{\mathrm{ns}}$ & 21,4 & $0,77^{\mathrm{ns}}$ & 11,2 & $0,25^{\mathrm{ns}}$ & 40,6 \\
\hline $\mathrm{C}$ & $0,77 *$ & 46,0 & $0,74^{\mathrm{ns}}$ & 27,0 & $1,20^{\mathrm{ns}}$ & 30,3 \\
\hline $\mathrm{D}$ & $-0,17^{\text {ns }}$ & 9,0 & $0,42^{\mathrm{ns}}$ & 10,1 & $1,12^{\mathrm{ns}}$ & 27,0 \\
\hline Geral & $0,37 * * *$ & 65,8 & $0,39 * *$ & 49,3 & $0,65 * * *$ & 69,3 \\
\hline
\end{tabular}

nsNão-significativo. *,** e ***Significativo a 5,1 e $0,1 \%$, respectivamente.

Tabela 4. Critério para classificação do tipo de rebanho bubalino da raça Mediterrâneo no Brasil.

\begin{tabular}{lcccc}
\hline $\begin{array}{l}\text { Tipo de } \\
\text { rebanho }\end{array}$ & $\begin{array}{c}\text { Utilizam } \\
\text { reprodutores } \\
\text { externos }\end{array}$ & $\begin{array}{c}\text { Utilizam os } \\
\text { próprios } \\
\text { reprodutores }\end{array}$ & $\begin{array}{c}\text { Vendem } \\
\text { reprodutores }\end{array}$ & $\begin{array}{c}\text { Tipo do rebanho } \\
\text { utilizado no } \\
\text { estudo }\end{array}$ \\
\hline Núcleo & Não & Sim & Sim & 0 \\
Multiplicador 1 & Sim & Sim & Sim & 3 \\
Multiplicador 2 & Sim & Não & Sim & 0 \\
Comercial 1 & Sim & Sim & Não & 1 \\
Comercial 2 & Sim & Não & Não & 0 \\
Isolado & Não & Sim & Não & 0 \\
\hline
\end{tabular}

Assim, os ganhos obtidos, até este ano, foram decorrentes de melhoramento empírico baseado exclusivamente no fenótipo.

De acordo com Euclides Filho et al. (2000), tendências genéticas próximas de zero e até mesmo negativas, em bovinos, não são incomuns, principalmente quando resultam de avaliações conduzidas com uso de dados provenientes de rebanhos comerciais cujos critérios de seleção não são bem definidos, e, principalmente, não são uniformes.

Considerando-se intensidade de seleção de 1,24 (ou seja, retenção de $5 \%$ dos machos e $75 \%$ das fêmeas), e as herdabilidade diretas e os desvios-padrão fenotípicos de P205, P365 e P550, e o intervalo de geração dos rebanhos (11,3 anos), o progresso genético esperado $(\Delta \mathrm{G})$, por ano, seria da ordem de 1,54, 2,64 e 4,88 kg, correspondendo a $0,78,0,87$ e $1,14 \%$ da média da população para P205, P365 e P550. É importante frisar que se o intervalo de geração fosse reduzido pela metade (aproximadamente 5,5 anos), os possíveis ganhos genéticos, por ano, seriam duplicados.

As tendências (regressão linear) fenotípica e ambiental para P205 não foram significativas ( $>0,05)$. Esse resultado, possivelmente, é explicado pelo fato de que, no período do nascimento ao desmame, o ambiente materno exerce grande influência sobre o bezerro (Fries \& Albuquerque, 1998), e muitas matrizes, além de amamentarem a prole, são ordenhadas com finalidade da comercialização do leite e derivados.
O peso aos 365 dias de idade apresentou tendências (regressão linear) fenotípica e ambiental significativas ( $\mathrm{p}<0,01$ ) e iguais a 5,62 e 4,42 $\mathrm{kg} \mathrm{ano}^{-1}$, respectivamente. As regressões lineares para P550 também foram significativas $(p<0,01)$, com valores de 10,53 e $10,39 \mathrm{~kg} \mathrm{ano}^{-1}$ para o progresso fenotípico e ambiental, respectivamente.

As tendências fenotípicas equivaleram a 2,09 e 3,09\% do incremento no ganho anual nos pesos aos $365 \mathrm{e}$ 550 dias de idade. Este resultado evidencia a melhora do ambiente de criação dos animais na fase pósdesmama.

\section{Conclusões}

1. Há potencial para seleção na população estudada no que se refere a efeitos diretos estimados, para as três características.

2. Para aumento nos ganhos genéticos, são necessários a redução do intervalo de geração, o aumento do tamanho efetivo, o uso de reprodutores avaliados com base nos valores genéticos preditos pelo BLUP e o controle dos acasalamentos de animais aparentados.

\section{Agradecimentos}

À Universidade Estadual do Sudoeste da Bahia, à Fundação de Amparo à Pesquisa do Estado da Bahia, ao Conselho Nacional de Desenvolvimento Científico e Tecnológico e ao Grupo Pagab (Programa de Avaliação Genética Animal da Bahia), pelo apoio financeiro.

\section{Referências}

BOLDMAN, K.G.; KRIESE, L.A.; VAN VLECK, L.D.; VAN TASSEL, C.P.V.; KACHMAN, S.D. A manual for use of MTDFREML: a set of programs to obtain estimates of variances and covariances [DRAFT]. Lincoln: Department of Agriculture, Agricultural Research Service, 1995. 120p. 
CARNEIRO, P.L.S.; MALHADO, C.H.M.; EUCLYDES, R.F.; TORRES, R.A.; LOPES, P.S.; CARNEIRO, A.P.S.; CUNHA, E.E. Oscilação genética em populações submetidas a métodos de seleção tradicionais e associados a marcadores moleculares. Revista Brasileira de Zootecnia, v.35, p.84-91, 2006.

CUNHA, E.E.; EUCLIDES, R.F.; TORRES, R.A.; CARNEIRO, P.L.S. Simulação de dados para avaliação genética de rebanhos de gado de corte. Arquivo Brasileiro de Medicina Veterinária e Zootecnia, v.58, p.381-387, 2006.

EUCLIDES FILHO, K.; SILVA, L.O.C.; ALVES, R.G.O.; FIGUEREIDO, G.R. Tendência genética na raça Gir. Pesquisa Agropecuária Brasileira, v.35, p.787-791, 2000.

FARIA, F.J.C.; VERCESI FILHO, A.E.; MADALENA, F.E.; JOSAHKIAN, L.A. Estrutura populacional da raça Nelore Mocho. Arquivo Brasileiro de Medicina Veterinária e Zootecnia, v.54, p.501-509, 2002.

FARIA, F.J.C.; VERCESI FILHO, A.E.; MADALENA, F.E.; JOSAHKIAN, L.A. Parâmetros populacionais do rebanho Gir Mocho registrado no Brasil. Revista Brasileira de Zootecnia, v.30, p.19841988, 2001. (Suplemento).

FERRAZ FILHO, P.B.; RAMOS, A.A.; SILVA, L.O.C.; SOUZA, J.C.; ALENCAR, M.M.; MALHADO, C.H.M. Tendência genética dos efeitos diretos e maternos sobre os pesos à desmama e pósdesmama de bovinos da raça Tabapuã no Brasil. Revista Brasileira de Zootecnia, v.31, p.635-640, 2002.

FRIES, L.A.; ALBUQUERQUE, L.G. Pressuposições e restrições dos modelos animais com efeitos maternos em gado de corte. In: COSTA, M.J.; CROMBERG, V.U. Comportamento materno em mamíferos: bases teóricas e aplicações aos ruminantes domésticos. Jaboticabal: SBEt-ETCO, 1998. p.179-214.

GIANNOTTI, J.G.; PACKER, I.U.; MERCADANTE, M.E.Z. Meta-análise das estimativas de herdabilidade para características de crescimento em bovinos de corte. Revista Brasileira de Zootecnia, v.34, p.1173-1180, 2005.

GODDARD, M.G.; SMITH, C. Optimum number of bull sires in dairy cattle breeding. Journal of Dairy Science, v.73, p.1113$1122,1990$.

GUTIERREZ, J.P.; GOYACHE, F. A note on ENDOG: a computer program for analysing pedigree information. Journal of Animal Breeding and Genetics, v.122, p.172-176, 2005.
JORGE, A.M.; ANDRIGHetTO, C.; CASTro, V.S. Desenvolvimento ponderal de bubalinos da raça Murrah criados em pastagem de Brachiaria brizantha no Centro-Oeste do Estado de São Paulo, Brasil. Ciência Rural, v.35, p.417-421, 2005.

LÔBO, R.N.B.; MADALENA, F.E.; VIEIRA, A.R. Average estimates of genetic parameters for beef and dairy cattle in tropical regions. Animal Breeding Abstracts, v.68, p.433-462, 2000.

MALHADO, C.H.M. Análise fenotípica e genética do desenvolvimento ponderal de bubalinos de corte no Brasil. 2005. 189p. Tese (Doutorado) - Universidade Estadual Paulista, Botucatu.

MALHADO, C.H.M.; RAMOS, A.A.; CARNEIRO, P.L.S.; SOUZA, J.C.; PICCININ, A. Parâmetros e tendências da produção de leite em bubalinos da raça Murrah no Brasil. Revista Brasileira de Zootecnia, v.36, p.376-379, 2007.

MEUWISSEN, T.H.E.; WOOLLIAMS, J. Effective sizes of livestock populations to prevent a decline in fitness. Theoretical and Applied Genetics, v.89, p.1019-1026, 1994.

MUIR, W.M. The interaction of selection intensity, inbreeding depression, and random genetic drift on short and long-term response to selection: results using finite locus and finite population size models incorporating directional dominance. American Society of Animal Science, v.79, p.1-11, 2000.

OLIVEIRA, J.A.; LÔBO, R.B.; NUNES, H.N. Tendência genética em pesos e ganhos de pesos em bovinos da raça Guzerá. Pesquisa Agropecuária Brasileira, v.30, p.1355-1360, 1995.

RAMOS, A.A.; MALHADO, C.H.M.; CARNEIRO, P.L.S.; AZÊVEDO, D.M.M.R.; GONÇALVES, H.C. Caracterização fenotípica e genética da produção de leite e do intervalo entre partos em bubalinos da raça Murrah. Pesquisa Agropecuária Brasileira, v.41, p.1261-1267, 2006.

RAMOS, A.A.; WECHSLER, F.S.; VAN ONSELEN, V.J.; GONCALVES, H.C. PROMEBUL: sumário de touros bubalinos. Botucatu: Unesp, 2002. 39p.

SAS INSTITUTE. Statistical Analysis System: user guide. Cary: SAS, 1999.

VERCESI FILHO, A.E.; FARIA, F.J.C.; MADALENA, F.E.; JOSAHKIAN, L.A. Estrutura populacional do rebanho Tabapuã registrado no Brasil. Arquivo Brasileiro de Medicina Veterinária e Zootecnia, v.54, p.609-617, 2002.

Recebido em 26 de setembro de 2007 e aprovado em 11 de fevereiro de 2008 